RHS. Revista. Humanismo. Soc. $7(1)$ : 52-68, junio 2019

\section{Calidad de vida laboral en docentes de una institución educativa en la ciudad de Medellín en el año 2018}

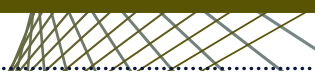

Quality of working life among teachers of an educational institution in the city of Medellin in 2018
Dora Aidé Ramírez González

darpsico@hotmail.com

https://doi.org/10.22209/rhs.v7n1a04

Recibido: febrero 27 de 2019

Aceptado: mayo 31 de 2019.

\section{Resumen}

El artículo tiene como propósito la descripción de la calidad de vida laboral a través de la medición de la percepción sobre las condiciones de trabajo, organización y demandas del trabajo, la motivación, los valores, el engagement, el estrés y el síndrome de burnout. La investigación es un estudio de caso con método cuantitativo y un enfoque empírico analítico conforme al diseño transeccional descriptivo. El universo poblacional estuvo conformado por 24 docentes de una institución educativa pública de Medellín en el año 2018, a los cuales se les aplicó el Cuestionario Internacional de Calidad de Vida Laboral de Organizaciones de Servicios Humanos. Se encontró que los dominios evaluados acorde con la calidad de vida laboral no presentan condiciones bajas o sin condiciones. Las conclusiones destacan la necesidad de gestionar de manera preventiva los dominios con sus dimensiones e ítems que aparecen en

1 Docente de la Escuela de Ciencias de la Salud, Universidad Nacional Abierta y a Distancia. Docente de la Facultad de Ciencias de la Salud, Fundación Universitaria María Cano. condiciones aceptables con necesidad de mejora para que no se constituyan en factores de riesgo para la calidad de vida laboral en la institución.

Palabras clave: Calidad de vida laboral; Docente de secundaria; Factor de riesgo; Institución estatal de educación; Percepción del entorno; Sector público.

\section{Abstrac}

This paper aims to describe the quality of working life by measuring the perception of working conditions, organization and demands of work, motivation, values, engagement, stress and the burnout syndrome. This is a case study with a quantitative method and an analytical empirical approach according to the descriptive transectional design. The population universe consisted of 24 teachers from a public education institution in Medellin, to whom the International Questionnaire on Quality of Work Life of Human Services Organizations was administered in 2018. As part of the findings of the research study, the domains assessed to establish the quality of working life yielded neither no nor low conditions. The conclusions highlight the need to preventively manage the domains with their dimensions and items that appear in acceptable conditions with a need for improvement, so they do not become risk factors for the quality of working life in the institution.

Keywords: Quality of working life; high school teacher; risk factor; state institution of education; perception of the environment; public sector. 


\section{Introducción}

$\mathrm{P}$ ara las instituciones educativas es necesario identificar las posibles condiciones de riesgo a las cuales están expuestos los docentes por la actividad laboral que desempeñan. Sobre todo, en el contexto de la nueva gestión pública que se propone mejorar el desempeño del sector público para fortalecer la tarea del Estado, al tener en cuenta logros acumulados de experiencias exitosas del ámbito privado de décadas pasadas, donde incorpora la gestión empresarial privada a lo público, el cual enfatiza en indicadores orientados a resultados de rentabilidad, competencia, y crecimiento, como parece sugerir López en su análisis sobre la nueva gestión pública (sf, p. 5).

Las transformaciones en el mundo del trabajo afectan la calidad de vida laboral, dada la flexibilización de las políticas laborales en un contexto neoliberal que permea toda la economía, incidiendo incluso en lo público y, por supuesto, en el privado que es el que jalona la economía capitalista.

De ahí la necesidad de conocer los factores de riesgo en las Instituciones Educativas, dado que pueden generar desequilibrios en los docentes y tener graves repercusiones para la salud ocupacional por las demandas laborales del oficio que realizan, «en un contexto cifrado por la mercantilización de lo existente en función de un sistema político-económico imperante, que sin duda ha permeado y dominado las lógicas actuales en la prestación de servicios educativos» (Ramírez González y Soler Gaona, 2015, p. 118).

Específicamente, la Institución Educativa no cuenta con un diagnóstico de calidad de vida laboral, de ahí la necesidad de identificar las posibles condiciones de riesgo a las cuales están expuestos los docentes, sobre todo en el contexto de la nueva gestión pública dada su condición de institución pública.

El presente artículo tiene como propósito la descripción de la calidad de vida laboral a través de la medición de la percepción sobre 1) las tendencias en el mundo del trabajo, 2) las condiciones de trabajo, 3) la organización del trabajo, 4) las demandas del trabajo, 5) la motivación, 6) los valores, 7) el engagement, 8) el estrés, y 9) el síndrome de burnout. Estos nueve dominios son el centro del estudio sobre calidad de vida laboral.

El proceso metodológico utilizado en la investigación es un estudio de caso, con un enfoque empírico analítico y alcance descriptivo, se utilizó el Cuestionario Internacional de Calidad de Vida Laboral en Organizaciones de Servicios Humanos.

Se encontró que de los 9 dominios constituidos por 27 dimensiones y estas por 175 ítems, se encuentran en condiciones aceptables y con necesidad de mejorar los dominios de condiciones de trabajo, organización del trabajo, estrés y síndrome de burnout, por su parte, los dominios de demandas del trabajo, tendencias del mundo del trabajo, valores, motivación y engagement, se encuentran en buenas condiciones.

Las conclusiones centrales redundan en la relevancia de gestionar de manera preventiva los dominios con sus dimensiones e ítems que aparecen en condiciones aceptables con necesidad de mejora para que no se constituyan en factores de riesgo para la calidad de vida laboral en la institución. 


\section{Metodología}

El presente artículo se compromete con un alcance descriptivo al conocer características y manifestaciones asociadas a la calidad de vida laboral según la experiencia docente en una institución educativa de la ciudad de Medellín. La investigación es cuantitativa, en la medida que retomó las categorías enmarcadas en la recolección, procesamiento y análisis de los dominios, dimensiones e ítems relacionados en el Cuestionario Internacional de Calidad de Vida Laboral, en el cual se pretendió reconocer los componentes principales de la calidad de vida laboral de los docentes de la Institución Educativa estudiada.

El enfoque es el empírico analítico. Se recolectan y analizan los datos para diagnosticar la calidad de vida laboral acorde con las condiciones de trabajo a los cuales están expuestos los docentes. En esta forma de hacer conocimiento la realidad queda expresada en la respuesta al cuestionario de los participantes en la investigación.

La investigación es un estudio de caso, debido a que los resultados solo tienen validez particular y no se generalizan a otras instituciones; en este sentido, dicho estudio "consiste en la recopilación e interpretación detallada de toda información posible sobre un individuo, una institución, una empresa, un movimiento social particular» (Vieytes, 2009, p. 59). La información se recogió en un momento único y acordado con la Institución, según al diseño transeccional descriptivo señalado por Hernández Sampieri, Fernández Collado, y Baptista Lucio (2014, pp. 155-156), para indagar por las condiciones de trabajo relacionadas con la calidad de vida laboral.
Como fuentes de información se tuvieron los 24 docentes encuestados, que fue universo poblacional para la institución, así como también documentos de consulta en temas relativos al objeto de estudio sobre calidad de vida laboral: libros, artículos de revistas, capítulos de libros, la normatividad existente (decretos, leyes), etc., citados en el presente artículo. También tuvimos las respectivas notas y documentos de clase correspondientes a los módulos de la especialización en salud ocupacional, así mismo, el documento de trabajo sobre glosario de términos (texto que se cita pero no se referencia por ser material de trabajo).

La técnica aplicada fue el cuestionario, de acuerdo con Hernández Sampieri, Fernández Collado, y Baptista Lucio, «[...] consiste en un conjunto de preguntas respecto a una o más variables a medir» $(2014$, p. 217). En este sentido, se tuvo en cuenta el cuestionario sobre Calidad de Vida Laboral en Organizaciones de Servicios Humanos, diseñado por Blanch, Sahagún, Cantera, y Cervantes (2010, pp. 157-170), del cual solo se tuvieron en cuenta los primeros 11 puntos, dado que es en estos ítems que se posibilita el abordaje de los 9 dominios desde un enfoque cuantitativo, los demás ítems son valoraciones personales sobre la experiencia laboral como docentes de la institución.

Este instrumento analiza la calidad de vida laboral en las organizaciones de servicios humanos a través de 9 dominios (condiciones de trabajo, organización del trabajo, demandas del trabajo, valores, tendencias del mundo del trabajo, motivación, estrés, síndrome de burnout y engagement); 27 dimensiones que se encuentran en el interior de los dominios, y 175 ítems que están adscritos a las dimensiones. (Ver Tabla 1). 
Tabla 1. Categorías para el análisis cuantitativo.

\begin{tabular}{|c|c|c|}
\hline DOMINIOS & DIMENSIONES & No. ITEMS \\
\hline \multirow{4}{*}{$\begin{array}{l}\text { CONDICIONES DEL TRABAJO } \\
\text { (ambientales, relaciones con los } \\
\text { colaboradores y entorno físico) }\end{array}$} & Entorno físico del trabajo & 4 ítems \\
\hline & Relaciones con los colaboradores & 3 ítems \\
\hline & Control y autonomía sobre el trabajo & 10 ítems \\
\hline & Relaciones con jefes y directivos & 4 ítems \\
\hline ORGANIZACIÓN DEL TRABAJO & Organización del trabajo & 15 ítems \\
\hline DEMANDAS DEL TRABAJO & Demandas del trabajo & 12 ítems \\
\hline \multirow[t]{6}{*}{ VALORES } & Relación estable de la interacción humana & 3 ítems \\
\hline & Autorrealización & 3 ítems \\
\hline & Bienestar social del trabajo & 9 ítems \\
\hline & Rentabilidad & 2 ítems \\
\hline & Complacencia profesional & 10 ítems \\
\hline & Razón moral del trabajo & 10 ítems \\
\hline \multirow[t]{5}{*}{ TENDENCIAS EN EL MUNDO DEL TRABAJO } & Estructura mercantil del trabajo & 8 ítems \\
\hline & conductismo normativo & 4 ítems \\
\hline & Autonomía moral & 4 ítems \\
\hline & Ética situacional & 3 ítems \\
\hline & Pragmatismo utilitarista & 4 ítems \\
\hline \multirow[t]{2}{*}{ MOTIVACIÓN } & Satisfacción laboral & 18 ítems \\
\hline & Promoción y seguridad en el trabajo & 4 ítems \\
\hline \multirow[t]{3}{*}{ ESTRÉS } & Salud general & 5 ítems \\
\hline & Vitalidad & 4 ítems \\
\hline & Salud mental & 4 ítems \\
\hline \multirow[t]{3}{*}{ SÍNDROME DE BURNOUT } & Cansancio emocional & 6 ítems \\
\hline & Falta de realización personal & 7 ítems \\
\hline & Despersonalización & 2 ítems \\
\hline \multirow[t]{2}{*}{ ENGAGEMENT } & Vigor y dedicación & 10 ítems \\
\hline & Absorción & 7 ítems \\
\hline
\end{tabular}

Fuente: Elaboración propia a partir de Blanch, Sahagún, Cantera, y Cervantes (2010).

Para la presentación de resultados y discusión se partió del baremo para los dominios y para las dimensiones, una escala para los ítems, y la validez del modelo a través del análisis de varianza con un valor menor de 0.05, lo cual refleja la percepción que tienen los docentes sobre las condiciones de calidad de vida laboral experimentadas. Los baremos para dominios y las dimensiones oscilaron entre los siguientes cinco rangos posibles: sin condiciones (0.00-20.99), bajas condiciones (21.00-40.99), condiciones aceptables, pero 
con necesidad de mejora (41.00-60.99), condiciones buenas (61.00-80.99), y condiciones óptimas (81.00-100.00). El baremo es el criterio de valoración cuantitativo.

\section{Resultados y discusión}

En las instituciones públicas que ofrecen servicios humanos, como es el caso de la educación, se torna crucial que se hagan mediciones sobre la percepción que tienen los docentes de la calidad de vida laboral en su quehacer actual, de tal manera que se pueda lograr el entendimiento de cómo se comporta la nueva gestión pública y cómo esta se encuentra impactada por los modelos neoliberales de la economía capitalista, la globalización, y la producción.

En este orden de ideas, las reformas educativas en Colombia procuran responder a exigencias de un entorno mundializado, como lo es la Ley General de Educación (Ley 115 de 1994) que entiende la educación como un servicio y las demás reglamentaciones que la secundan respondiendo a la Declaración de Bolonia (1999) que concibe la educación como un servicio que debe responder a los estándares de las nuevas economías y del entorno empresarial.

Lo anterior denota la necesidad de explorar la calidad de vida laboral, dadas las transformaciones en los entornos organizacionales acorde con estas nuevas exigencias del mercado, tal como lo exponen Segurado Torres y Agulló Tomás:

La preocupación por la calidad de la vida en el trabajo (CVL) cobra un especial interés en la década de los años 70 en los EE. UU., donde alcanza el reconocimiento social e institucional gracias a las actuaciones del «Movimiento de CVL". Las reivindicaciones de este nuevo movimiento parten de la necesidad de humanizar el entorno de trabajo prestando especial atención al desarrollo del factor humano y a la mejora de su calidad de vida (2002, p. 828).

Es así como la necesidad de humanizar el trabajo cobra relevancia, dado que además de afectarse las condiciones de trabajo se han generado nuevos fenómenos en detrimento de la calidad de vida laboral como lo es de manera notoria el estrés laboral, que se constituye en una problemática de salud pública de índole mundial y nacional, como lo expone la Organización Internacional del Trabajo en el referido informe sobre Estrés en el trabajo, donde menciona la prevalencia del estrés en relación con el trabajo en todo el mundo (2016); así mismo, sucede en Colombia de acuerdo con la primera y segunda encuesta nacional de condiciones de salud y de trabajo en el año 2007 y 2015, respectivamente, donde se evidencia la problemática del estrés en los entornos laborales, así mismo, la prevalencia de factores psicosociales en el trabajo que se pueden constituir en factores de riesgo o factores protectores para la salud y calidad de vida en el trabajo.

Es así, en el contexto de lo referido, que se realiza esta investigación sobre la percepción de la calidad de vida laboral en docentes de una institución básica y media, entendiendo que

el objetivo primordial de la CVL estriba en alcanzar una mayor humanización del trabajo a través del diseño de puestos de trabajo más ergonómicos, unas condiciones de trabajo más seguras y saludables, y unas organizaciones eficaces, más democráticas y participativas capaces de satisfacer las necesidades y demandas de sus miembros además de ofrecerles oportunidades de desarrollo profesional y personal (Segurado Torres y Agulló Tomás, 2002, p. 831). 
En la Institución Educativa se encontró que los dominios evaluados, según la calidad de vida laboral (condiciones de trabajo, organización del trabajo, demandas del trabajo, valores, tendencias en el mundo del trabajo, motivación, estrés, síndrome de burnout y engagement), no presentan condiciones bajas o sin condiciones, siendo este un punto relevante dado que no se evidenció en la totalidad de dominios, dimensiones e ítems un deterioro total y comprobado de las mismas y, por ende, la existencia de factores de riesgo actuales que sea necesario intervenir de manera prioritaria.

En los dominios de condiciones de trabajo, organización del trabajo, estrés y síndrome de burnout se constatan condiciones aceptables con necesidad de mejora, lo que indica que es necesario que se intervengan de manera preventiva estos dominios para que no se constituyan en factores de riesgo para la calidad de vida laboral.

Por su parte, en los dominios de demandas del trabajo, valores, tendencias en el mundo del trabajo, motivación y engagement se evidencian condiciones buenas, es decir, que estas condiciones son en la actualidad factores protectores en su generalidad y, por tanto, requieren ser promovidos para su sostenimiento a favor de la salud y calidad de vida laboral.

\section{Dominio: condiciones de trabajo}

En este orden de ideas, las condiciones de trabajo para efectos de este análisis se entenderán como lo expone la Batería de instrumentos del Ministerio de la Protección Social y la Pontifica Universidad Javeriana como las «características del trabajo y de su organización que influyen en la salud y bienestar del individuo» (2010, p. 20) y son factores presentes en el entorno laboral.
En ese sentido, se encontró que los docentes perciben estas condiciones aceptables, es decir, que presentan elementos favorables y a la vez de necesidad de mejoramiento. En el orden de prioridad en la intervención preventiva de acuerdo con los puntajes obtenidos se encuentra lo siguiente:

En cuanto a las relaciones con jefes y directivos (47.7) es necesario cualificar la posibilidad que tienen los docentes de participar en las decisiones organizacionales, en el mejoramiento de las relaciones con la dirección, en la evaluación que se hace del rendimiento profesional así como en el apoyo que se recibe del personal directivo, esta dimensión cobra relevancia dada la importancia de «[...] las interacciones que se establecen entre jefes y directivos y sus colaboradores susceptibles de mediar el equilibrio entre los procesos y fines de la organización y las necesidades y expectativas del trabajador» (Ramírez González y Soler Gaona, 2015, p. 51).

Con relación al entorno físico del trabajo (50.1) se hace necesario mejorar el entorno físico, instalaciones y equipamiento de trabajo, los recursos materiales y técnicos, la prevención de riesgos laborales y los servicios auxiliares como limpieza, restauración, seguridad, etc. Estas condiciones físicas pueden constituirse en factores de riesgo, dado que pueden generar accidentes de trabajo o afectación de la salud del docente a corto o largo plazo, así mismo, se puede afectar el desempeño laboral debido a la imposibilidad de acceder a estas condiciones que permiten un trabajo efectivo.

Al respecto de la dimensión de control y autonomía sobre el trabajo (60.75), se espera una revisión de la «posibilidad que el trabajo ofrece al individuo para influir y tomar decisiones sobre los diversos aspectos que intervienen en su 
realización» (Ministerio de la Protección Social y Pontifica Universidad Javeriana, 2010, p. 20). En este caso es necesario evaluar la posibilidad de los docentes de tomar decisiones en cuanto al tiempo de trabajo (horarios, ritmos, descansos, etc.), organización general de su trabajo, retribución económica, la carga de trabajo asignada, la posibilidad de conciliar trabajo - vida privada y familiar, la autonomía en la toma de decisiones profesionales, la justicia en la contratación, la remuneración y la promoción, las oportunidades para la formación continua y las vías de promoción laboral, es de anotar, que algunos de estos tópicos son estipulados desde el sistema educativo y no dependen directamente de la institución, por tal razón, se podrían abordar aquellos tópicos que estén a su alcance.

Por su parte, las relaciones con los colaboradores (76.11) se encuentran en condiciones buenas, lo que indica que este puede ser un factor protector para la calidad de vida laboral, dado que los docentes perciben que en el grupo de trabajo hay respeto, compañerismo y en algunas ocasiones reconocimiento del propio trabajo por colegas, dado que este último tópico se encuentra en condiciones aceptables.

\section{Dominio: organización del trabajo}

Los nuevos movimientos mundiales y macroeconómicos han generado nuevas formas de concebir y organizar el trabajo en función de la competitividad y productividad, en este sentido, el sector educativo también se ciñe a estas nuevas formas de gestión administrativa, en particular a la nueva gestión pública que es objeto de análisis en esta investigación. Como lo exponen Oliveira, Gonçalves y Melo Savana, estas reformas educativas introdujeron en consecuencia «[...] cambios que representan una real intensificación del trabajo de los maestros, reflejada en la sobrecarga provocada por los nuevos procesos de enseñanza y evaluación, obligándoles a encontrar medios alternativos de respuesta a las crecientes demandas» (2004, p. 189).

Esta nueva forma de organización del trabajo impacta también las instituciones educativas públicas, dado que ahora hacen parte del sector empresarial y su filosofía; es así como el

Sector empresarial se ha visto en la necesidad de reorientar los procesos organizacionales en la producción, en las relaciones de personal y en el uso de tecnología, para obtener una organización del trabajo con capacidad de adaptación y respuesta a los cambios de la economía global, lo que ha conllevado a analizar las nuevas formas organizativas del trabajo y su influencia en la flexibilización, y en la precariedad de las relaciones laborales (Useche y Parra Queipo, 2002, p. 69).

En ese sentido, se encontró que los docentes perciben la actual organización del trabajo en su institución en condiciones aceptables con necesidad de mejora (57.3), es decir, que la actual organización del trabajo responde de manera media a sus intereses, necesidades, a sus aspiraciones, a sus valores, al desarrollo de sus competencias profesionales, a la exigencia según sus capacidades, a sus expectativas, a la valoración con justicia de sus méritos, al estímulo de su compromiso laboral, a realizar un trabajo con gusto y motivación, con sensación de libertad, de crecimiento personal y sensación de ser útil en su trabajo. 


\section{Dominio: demandas del trabajo}

De acuerdo con el Ministerio de Protección Social y la Pontificia Universidad Javeriana, las demandas del trabajo hacen referencia «[...] a las exigencias que el trabajo impone al individuo. Pueden ser de diversa naturaleza, como cuantitativas, cognitivas o mentales, emocionales, de responsabilidad, del ambiente físico laboral y de la jornada de trabajo» (2010, p. 20).

Por lo tanto, se entenderá que el trabajo de los docentes de la Institución educativa en cuestión, enfrenta a esta población a unas exigencias y demandas propias de su labor que ameritan que los docentes las afronten y dispongan de sus propios recursos para equilibrarse y dar respuesta efectiva a las mismas y que dichas condiciones de trabajo favorezcan que estas demandas del trabajo no desborden el equilibrio y salud de los docentes.

Al respecto, en el dominio y dimensión de demandas del trabajo se encuentra en condiciones buenas (71.76), lo que indica que los docentes en términos generales logran un equilibrio entre las demandas del trabajo y sus propios recursos internos. Ellos se adaptan de manera óptima a las políticas de la institución, asumen los valores de la dirección, aceptan que les digan cómo hacer su trabajo, interiorizan las normas establecidas en su servicio, aplican el protocolo ante cualquier dilema de conciencia, se ajustan a los tiempos y ritmos de trabajo fijados, sin embargo, exponen que en términos medios logran identificarse con el espíritu de los cambios propuestos y con relación a su trabajo logran en términos medios o que en ocasiones se cumpla o en otras no, terminar en su jornada de trabajo lo que debían hacer, disponer del tiempo suficiente para hacer bien todo lo que deben hacer, se acumulan tareas pendientes a lo largo de la semana, llevarse tareas laborales a casa, y en fines de semana y vacaciones terminar trabajo atrasado.

\section{Dominio: valores}

En los contextos organizacionales actuales se ha generado una resignificación de los valores que priman en la prestación de un servicio, en particular en un servicio de carácter público como lo es la educación. Estos nuevos valores también tienen afectación en la humanización o deshumanización de las instituciones, dado que en todo caso priman los valores impuestos por el modelo económico capitalista de producción, de rentabilidad económica y competitividad o los valores propios del servicio como bien común, satisfacción de las personas que reciben el servicio y aquellos que lo proporcionan como es el caso de los docentes.

Es así como la nueva gestión pública presenta incoherencias, en cuanto a

[...] la plena vigencia de valores fundamentales tales como la imparcialidad, la justicia, la equidad, y el derecho de un procedimiento justo, como tareas propias de toda administración pública en una democracia, ha sido objeto de profundos cuestionamientos, acordes con las nuevas implicancias que el término «interés público" posee hoy para la sociedad (López, sf, p. 9).

En ese sentido, se encontró que los docentes perciben el dominio de valores en condiciones buenas (80.96). Este es el puntaje más alto dentro de los nueve dominios estudiados, lo que indica en términos generales que los docentes perciben de manera favorable los valores en cuestión, los cuales es importante seguir 
sosteniendo y estimulando para que sigan siendo factores protectores de la calidad de vida laboral. En los siguientes párrafos se expondrán las dimensiones de este dominio de valores acorde con los puntajes obtenidos.

Al respecto de la relación estable de la interacción humana (87.63), entendida como la estabilidad emocional de orden afectivo y social requerida en la conciliación vida familiar - vida laboral (Documento de trabajo Glosario de Términos), los docentes exponen que los valores de la familia y el trabajo son fundamentales y que se encuentran en condiciones óptimas en cuanto son cruciales para su estabilidad emocional, afectiva y social, no obstante, exponen que el reconocimiento de su trabajo por personas usuarias se encuentra en condiciones aceptables con necesidad de mejora. En concreto, la dimensión de la relación estable de la interacción humana se encuentra en condiciones óptimas.

La dimensión de autorrealización (85), se encuentra también en condiciones óptimas, dado que los docentes exponen que la importancia que le imprimen a los valores de salud, dinero y tiempo libre es óptima, esto sugiere que los docentes valoran positivamente estos aspectos en relación con satisfacción de sus necesidades humanas.

En cuanto a la dimensión de bienestar social del trabajo (85.77), se encuentra en condiciones óptimas. Esto indica que los docentes perciben que la importancia de los valores en la gestión de los servicios humanos en su institución es óptima en cuanto se logra la calidad del servicio, el bien común, calidad de vida laboral, ética del servicio público, eficiencia, satisfacción de las personas atendidas, así mismo, la competitividad y la productividad.
Al respecto de la rentabilidad económica perciben que este atributo es importante en términos medios para la gestión de los servicios humanos en su institución.

En la dimensión de rentabilidad (57.7), que se refiere a la valoración del coste beneficio frente al trabajo (Documento de trabajo Glosario de Términos), se encuentra que los docentes exponen que el espíritu de negocio privado y análisis de coste-beneficio son aceptables con necesidad de mejora, lo que indica que perciben que la institución no centra el trabajo y servicio educativo en estos aspectos relacionados con la rentabilidad.

En la dimensión de complacencia profesional (72.73), que se refiere a la satisfacción y alegría que produce la profesión, se caracteriza por la seguridad, la tranquilidad, el bienestar, la confianza, la certidumbre y la claridad (Documento de trabajo Glosario de Términos), se encuentra que los docentes la perciben en condiciones buenas, en cuanto logran experimentar satisfacción y seguridad en su trabajo de manera óptima, aunque exponen que pueden presentar en ocasiones sentimientos contrarios como intranquilidad - tranquilidad, impotencia - potencia, malestar - bienestar, desconfianza - confianza, incertidumbre - certidumbre, confusión - claridad, desesperanza - esperanza, y dificultad - facilidad. Lo anterior indica que en términos generales los docentes tienen complacencia profesional, aunque en algunas ocasiones presenten sentimientos relacionados o contrarios a esta dimensión.

En la dimensión de razón moral del trabajo (85.29), que se refiere a la estructura emotiva y profesional, se representa la sensibilidad, el optimismo, la bondad, el éxito, la capacidad y la competencia (Documento de trabajo Glosario 
de Términos). En este componente se encuentra que los docentes la perciben en condiciones óptimas, en cuanto logran experimentar por su trabajo sensibilidad, racionalidad, competencia, moralidad, bondad, éxito, capacidad, optimismo, eficacia y utilidad de su trabajo.

\section{Dominio: tendencias en el mundo del trabajo}

En cuanto a las tendencias en el mundo del trabajo se ha expuesto de manera previa las orientaciones mundialistas que han generado trasformaciones en los contextos organizacionales y en el trabajo, conduciendo a la precarización del trabajo, como lo expone el autor Cornfield (2006, p. 122), al identificar problemas relacionados en el mundo del trabajo: 1) con la globalización económica, el advenimiento del neoliberalismo y la restructuración de la burocracia; 2) el tránsito de una economía manufacturera a una de servicios; y 3) desigualdad social, surgimiento de las identidades políticas, y los viejos y nuevos movimientos sociales.

En este orden de ideas, la Organización Internacional del Trabajo (OIT) fomenta «[...] el crecimiento de empresas sostenibles y, por lo mismo, el crecimiento inclusivo y resultados en materia de trabajo decente» (2017, p. 4). En este sentido, para la OIT el trabajo decente, inclusivo y sostenible es una tendencia laboral para crear más y mejores empleos en todo el mundo.

Al respecto, se encuentran tendencias en el mundo laboral que pauperizan el trabajo o lo exaltan y dignifican como propone la OIT, es por ello relevante investigar al respecto sobre estos acontecimientos en las instituciones públicas.
En la Institución Educativa estudiada se encuentra una percepción favorable por parte de los docentes en el dominio de tendencias en el mundo del trabajo (63.61), en este orden de ideas, se encuentran en condiciones buenas o favorables cuatro dimensiones de este dominio, excepto la dimensión de estructura mercantil del trabajo, estas dimensiones se detallan a continuación.

La dimensión estructura mercantil del trabajo (49.92) se entiende como la percepción de los docentes frente a la transformación social y del trabajo generado por el neoliberalismo económico. Este componente que se estructura por la valoración del trabajo como mercancía, la normalización de los factores de libre competencia y la competitividad (Documento de trabajo Glosario de Términos), se encuentra en condiciones aceptables con necesidad de mejora, lo que indica que los docentes consideran que las nuevas tendencias en el mundo del trabajo han impactado los servicios educativos en cuanto en ocasiones se entiende que competir es un modo natural de trabajar, que un servicio es bueno en la medida en que es un buen negocio, que sería irracional resistirse a la lógica de la productividad, que quien desee tener un buen servicio debe de pagarlo, que el mercado pone a cada cual donde merece estar, que la eficiencia va en contra de la calidad del trato profesional, que las condiciones de trabajo son contrarias a los valores de la profesión, y que la vocación profesional está reñida con el trabajo actual.

El dominio conductismo normativo (76.19), entendido como la transformación personal frente a las normas y valores que toma la organización, se representa por el cumplimiento 
de normas y estándares, la lealtad hacia la institución, así como la defensa de los intereses de la organización (Documento de trabajo Glosario de Términos). Este componente se encuentra en condiciones buenas, pues los docentes cumplen de manera óptima las normas de la organización con profesionalidad, la lealtad a la institución es el primer imperativo de la profesionalidad; así mismo, exponen que en diversas ocasiones y en otras no aplica que la profesionalidad exige atender con eficiencia cualquier demanda de la institución pues cada profesional debe defender en primer lugar los intereses de su organización.

Sobre la autonomía moral (68.45), como la disponibilidad o iniciativa del trabajador para poder elegir la planificación y desarrollo de su trabajo desde el razonamiento moral (Documento de trabajo Glosario de Términos), se ubica en condiciones buenas, dado que los docentes perciben que de manera óptima hacen uso de la ética profesional cuando compromete a cuestionar algunas pautas de la dirección y, así mismo, el considerar que la sobrecarga de trabajo deteriora la profesionalidad; no obstante, consideran que a veces el código ético obliga a enfrentarse a decisiones de la dirección, y que cuando la institución no da los medios suficientes, no hay porque cumplir las metas que impone.

En la dimensión ética situacional (69.44), entendida como las exigencias morales individuales y colectivas dadas por las pautas y las decisiones institucionales (Documento de trabajo Glosario de Términos), se ubica en el rango de condiciones buenas, dado que los docentes exponen que la escasez de medios se debe compensar con profesionalidad, en algunas situaciones la ética profesional obliga a desviarse de algunas pautas de la gerencia, en un conflicto de intereses entre organización y usuarios, a veces hay que aliarse con estos. Esto indica que los docentes aún son guiados en términos generales por sus exigencias morales que influyen en lo individual, así como en lo colectivo.

En la dimensión pragmatismo utilitarista (69.19), entendida como la valoración de las exigencias del trabajo hacia la prestación de servicios rentables, con calidad y eficiencia; es decir, evalúa la construcción de la racionalidad humana en orden a una acción productiva y eficiente (Documento de trabajo Glosario de Términos). Se ubica en el rango de condiciones buenas, dado que los docentes cuestionan que en ocasiones se deba dar una adaptación a la flexibilización laboral, a las decisiones que se deben tomar calculando coste-beneficio, al hecho de considerar que carece de sentido ofrecer un servicio no rentable y, por último, que se deban abandonar las prácticas ineficientes, dado que lo anterior parte de considerar que todo servicio debe ser rentable, eficiente, y adaptarse a las nuevas demandas del trabajo, las cuales se pueden cuestionar.

\section{Dominio: motivación}

En el dominio motivación se obtuvo un puntaje de (76), lo que indica que es una condición buena; es decir, que los docentes se sienten motivados en su trabajo partiendo de los factores extrínsecos e intrínsecos, los cuales contribuyen a su estado de ánimo y vinculación positiva con la realización de su trabajo. Tal como lo exponen Ramírez, Abreu, y Badii (2008, pp. 172-173), los factores de motivación están relacionados con factores de higiene y factores motivadores. Los primeros satisfacen las necesidades de las personas como motivación extrínseca, por ejemplo: el salario y los beneficios; la seguridad laboral o grado de confianza del trabajador 
sobre su continuidad en el empleo; las posibilidades de promoción, de cara a conseguir estatus laboral así como social; las condiciones de trabajo como horario laboral, las características del propio lugar de trabajo, instalaciones y materiales; el estilo de supervisión, o grado y forma de control de la organización sobre el contenido, la realización de la tarea que lleva a cabo un trabajador; el ambiente social del trabajo será facilitador de la motivación en tanto dé oportunidades de interacción con otras personas, proporcione feedback constructivo permitiendo la relación con el líder formal. Los segundos satisfacen las necesidades de las personas como motivación intrínseca, por ejemplo: la consecución de logros, las características de la tarea, la libertad así como la autonomía con respecto a la tarea, la implicación de conocimientos además de las habilidades, la retroalimentación, y el reconocimiento.

En este orden de ideas, se encuentra que en el dominio de motivación la dimensión de satisfacción laboral (78.47) se ubica en condiciones buenas, entendiendo esta como el grado de conformidad respecto a su entorno de trabajo, valora la motivación, el rendimiento personal y profesional, el sentido hacia el trabajo y la convicción de trabajar siguiendo el ordenamiento organizacional (Documento de trabajo Glosario de Términos). Es así como los docentes experimentan condiciones óptimas en cuanto a su motivación por el trabajo, la identificación con los valores de la organización, el rendimiento profesional, la capacidad de gestión de la carga de trabajo, la autoestima profesional, la confianza en el futuro profesional, el sentido del trabajo, el acatamiento de las pautas de la dirección, la satisfacción con el trabajo, la realización profesional, la eficacia profesional, el compromiso con el trabajo y la competencia profesional, así mismo, consideran que se debe mejorar la cordialidad en mi ambiente social de trabajo, la conciliación del trabajo con la vida privada, la calidad de vida laboral, el estado de ánimo laboral y el nivel de excelencia de mi organización.

La dimensión de la promoción y seguridad en el trabajo (64.88) se encuentra en condiciones buenas. Estas son las condiciones del entorno de trabajo que permiten generar buenas percepciones de seguridad, de calidad, de participación en la toma de decisiones y permiten establecer oportunidades de promoción laboral y calidad en las condiciones del trabajo (Documento de trabajo Glosario de Términos). Sobre esta dimensión los docentes exponen que experimentan que en los últimos años se ha logrado aumentar las sensaciones de seguridad en el trabajo, así mismo, el incremento medio de la calidad de las condiciones de trabajo, las oportunidades de promoción laboral y la participación en las decisiones de la organización, siendo importante continuar mejorando en las mismas.

\section{Dominio: estrés}

Como se ha mencionado de manera previa, el estrés se ha constituido en el contexto mundial y nacional en una problemática de salud que aqueja los diversos escenarios en que se desenvuelve el ser humano, el ámbito laboral no está exento de este fenómeno que es la base de accidentes de trabajo y enfermedades laborales que aumentan su cifra de manera exponencial. Partiendo de la Comisión Europea el estrés está relacionado con el trabajo y puede definirse como:

El conjunto de reacciones emocionales, cognitivas, fisiológicas y del comportamiento a ciertos aspectos adversos o nocivos del contenido, 
la organización o el entorno de trabajo. Es un estado que se caracteriza por altos niveles de excitación y angustia, con la frecuente sensación de no poder hacer frente a la situación (1999, p. 3).

En correspondencia con lo expuesto, se encuentra que el dominio de estrés obtiene el puntaje más bajo de los nueve dominios estudiados, lo cual es consistente con los resultados de las dimensiones que lo constituyen, estos puntajes corresponden a condiciones aceptables con necesidad de mejora.

En la dimensión salud general el puntaje obtenido es (55.74), indicando los docentes que en término medio se presentan los síntomas, es decir, en diversas ocasiones presentan síntomas que se expondrán acorde con su puntaje y mayor frecuencia, estos son: tensiones musculares, dolores de espalda, dolores de cabeza, insomnio y trastornos digestivos.

En la dimensión salud mental el puntaje obtenido es (47.04), indicando los docentes que, en término medio, es decir, en ocasiones, se presenta desgaste emocional, agotamiento físico, saturación mental, y sobrecarga de actividad laboral.

En la dimensión de vitalidad el puntaje obtenido es bueno con (66.31), entendido como un estado emocional de las personas que implica la presencia de vigor y energía para realizar de buena manera sus actividades personales y profesionales. Los docentes indican que, aunque en términos generales tienen vitalidad para la realización de sus labores, en ocasiones se presenta desgaste emocional, agotamiento físico, saturación mental, y sobrecarga de actividad laboral.

\section{Dominio: sindrome de burnout}

En correspondencia con los datos anteriores, el estrés se encuentra en condiciones aceptables y con necesidad de mejora, así mismo, el dominio de Síndrome de Burnout (58.33), lo que indica que los síntomas generados por el estrés laboral pueden ser un factor de riesgo potencial para que se genere los síntomas de un síndrome de burnout que se constituye como enfermedad laboral clasificada en la tabla de enfermedades laborales del Decreto 1477 de 2014; en este sentido, es importante aclarar que el síndrome de burnout presenta dos enfoques teóricos para su abordaje:

Para unos autores es considerado un tipo específico de estrés de los profesionales asistenciales producto de la demanda emocional generada por la interacción con gente y para otros es visto como la última fase del estrés laboral crónico. Se ha denominado también como síndrome de estar quemado o síndrome del estrés laboral asistencial o síndrome de desgaste profesional (Barbosa Ramírez, Muñoz Ortega, Rueda Villamizar, y Suárez Leiton, 2009, p. 22).

Al respecto, los docentes presentan en la dimensión de cansancio emocional (58.33) condiciones aceptables con necesidad de mejora. En la actualidad sienten en ocasiones agotamiento emocional por el trabajo, al final de la jornada, sienten que el trabajo los ha consumido, sienten fatiga por la mañana al enfrentarse a otro día de trabajo, sienten que trabajar todo el día realmente es estresante y sienten que en el trabajo se han quemado, no obstante, sienten que son capaces de resolver eficazmente los problemas surgidos en su trabajo. 
En la dimensión de despersonalización (44.44), con condiciones aceptables con necesidad de mejora, los docentes exponen que en ocasiones experimentan pérdida de interés desde que empezaron en el puesto actual de trabajo y que han perdido entusiasmo por el mismo, esto puede ser correspondiente o puede generar a futuro que los docentes desarrollen actitudes negativas e insensibles hacia los destinatarios del servicio que es como se entiende en su sentido pleno el concepto de despersonalización (Documento de trabajo Glosario de Términos).

En la dimensión falta de realización personal (72.24), con condiciones buenas, entendida esta como las respuestas negativas hacia el trabajo y la persona reflejadas en depresión, moral baja, evitación de las relaciones profesionales e interpersonales, baja productividad, y pobre autoestima (Documento de trabajo Glosario de Términos). En este sentido, los docentes experimentan de manera positiva que contribuyen eficazmente a la actividad de la organización, que hacen bien su trabajo y les estimula conseguir objetivos en este, sienten que realizan muchas cosas valiosas y tienen seguridad que son eficaces terminando sus labores, solo en ocasiones se preguntan con cinismo sobre la utilidad de su trabajo y dudan sobre el valor del mismo.

\section{Dominio: engagement}

El dominio de engagement está fuertemente relacionado con la motivación laboral, lo cual es correspondiente con los resultados obtenidos en el dominio de motivación. El engagement se encuentra en condiciones buenas (77.94), este se relaciona con la pasión por lo que se hace en el trabajo, con la conducta del trabajador y los procesos que desarrolla en la empresa haciendo del trabajo una experiencia positiva. En otras palabras, el engagement trata sobre la marcha óptima de las organizaciones acorde con la motivación de los trabajadores (Documento de trabajo Glosario de Términos).

Así mismo, se podría considerar que este dominio en conjunción con la motivación, pueden contribuir como factores protectores para la salud y calidad de vida de los docentes. Tal como lo exponen Salanova y Schaufelli, el engagement es «[...] un proceso de motivación positiva de mejora de la salud ocupacional, y contrario al proceso de deterioro de la salud que causa el burnout o síndrome de quemarse por el trabajo» (2009, p. 23).

En este dominio la dimensión de vigor y dedicación con 79.93, en condiciones buenas, se constituye en la fuerza, paciencia y perseverancia con la que disponen las personas para realizar de la mejor manera posible las actividades laborales y personales (Documento de trabajo Glosario de Términos). De ahí que los docentes expongan que en la actualidad en su trabajo sienten que tiene sentido y propósito, que son fuertes y vigorosos, sienten entusiasmo, se inspiran, se sientes orgullosos del trabajo que hacen, se sienten felices cuando están absortos en el trabajo, cuando se levantan por la mañana sienten ganas de ir a trabajar, y cuando están trabajando olvidan todo lo que pasa alrededor de ellos; no obstante, en algunas ocasiones y en otras no, sienten que están llenos de energía y que el tiempo «vuela» cuando están trabajando.

Por último, la dimensión de absorción que es el grado de involucramiento de la persona hacia el trabajo, bajo el cual, en algunos casos puede llevar a un horario de trabajo excesivo por la vinculación emocional de la persona hacia el mismo (Documento de trabajo Glosario de Términos), se encuentra en condiciones buenas 
con un puntaje de 75.09, donde los docentes hacen alusión a estar inmersos en su trabajo, considerar que es retador, son persistentes en él e Incluso cuando las cosas no van bien continúan trabajando; no obstante, exponen que en términos medios pueden continuar trabajando durante largos periodos de tiempo, se dejan llevar por el trabajo y se les hace difícil desconectarse del trabajo.

\section{Conclusiones}

Partiendo de los hallazgos y análisis realizados en esta investigación, donde se abordan 9 dominios que contienen 27 dimensiones y estas a su vez con 175 ítems, que permiten vislumbrar la percepción sobre la calidad de vida laboral de los docentes de la Institución Educativa, se centrarán las conclusiones en los resultados de los 9 dominios con sus dimensiones que son el centro de análisis y posterior intervención, los cuales son las condiciones en el trabajo, organización del trabajo, demandas del trabajo, valores, tendencias en el mundo del trabajo, motivación, estrés, síndrome de burnout, y engagement.

Los dominios y sus dimensiones estudiadas pueden constituirse en factores de riesgo o factores protectores de la calidad de vida laboral de los docentes y, por ende, de su salud y desempeño profesional. Es de anotar que aquellos que se encuentren en bajas condiciones o sin condiciones son los que requieren una intervención prioritaria, dado su posible riesgo; sin embargo, en la institución educativa no se encuentra ningún dominio y/o dimensiones en estos rangos, lo que es favorable para la institución e indica que ninguno de los tópicos evaluados se encuentra en condición de imperante intervención.
Se encuentra que los dominios de condiciones del trabajo, organización del trabajo, estrés y síndrome de burnout se perciben con condiciones aceptables con necesidad de mejora; esto es, que estos dominios en cuestión evidencian un punto de equilibrio medio, pero requieren ser intervenidos para que no se constituyan en factores de riesgo, pues, aunque cuentan con un mínimo necesario de bienestar al respecto, están dentro de sus expectativas y necesidades cualificarlas.

Es de anotar que la puntuación media con necesidad de mejora de estos dominios indica que sus dimensiones requieren ser intervenidas de manera preventiva, en específico, las siguientes: entorno físico del trabajo, control y autonomía sobre el trabajo, relaciones con jefes y directivos, organización del trabajo, salud general, salud mental, cansancio emocional, y despersonalización. En el caso de las dimensiones de relaciones con colaboradores, vitalidad y falta de realización personal se encuentran condiciones buenas, lo que indica que se pueden implementar acciones de promoción para que continúen siendo factores protectores.

En este orden de ideas, los dominios de demandas del trabajo, valores, tendencias del mundo del trabajo, motivación y engagement se encuentran en condiciones buenas, lo que amerita un sostenimiento y promoción de dichos dominios para que continúen siendo factores protectores de la calidad de vida laboral. Aun así, es importante que en las dimensiones de estos dominios que se encuentran en condiciones aceptables con necesidad de mejora se intervengan de manera preventiva, estas son en el dominio valores la rentabilidad y en el dominio tendencias del mundo del trabajo la dimensión estructura mercantil del trabajo, lo anterior, puede ser correspondiente con los cambios en la nueva gestión pública que han sido impactados 
por un modelo neoliberal y de productividad en las instituciones educativas.

En cuanto a los dominios mencionados con condiciones buenas (demandas del trabajo, valores, tendencias del mundo del trabajo, motivación y engagement), abarcan dimensiones que se encuentran en condiciones buenas $u$ óptimas. Estas son objeto de promoción para que continúen siendo factores protectores, tales como las demandas del trabajo, relación estable de la interacción humana, autorrealización, bienestar social del trabajo, complacencia profesional, razón moral del trabajo, conductismo normativo, autonomía moral, ética situacional, pragmatismo utilitarista, satisfacción laboral, promoción y seguridad en el trabajo, vigor y dedicación, y absorción.

Se logra con esta investigación el conocimiento y descripción de la percepción de la calidad de vida laboral de los docentes de la institución educativa, en la cual, sin duda, intervienen diversos tópicos de índole institucional pero también del macro sistema económico actual y del sistema educativo, siendo este un insumo para que en la institución se analice y definan aquellos aspectos que pueden ser intervenidos directamente según sus posibilidades y alcances.

\section{Referencias}

- Barbosa Ramírez, L. C; Muñoz Ortega, M. L; Rueda Villamizar, P. X; y Suárez Leiton, K. G. (2009). Síndrome de Burnout y estrategias de afrontamiento en docentes universitarios. Revista Iberoamericana de Psicología: Ciencia y Tecnología. 2(1), 21-30. Recuperado de https://bit.ly/2JYfp3o
- Blanch, J. M; Sahagún, M; Cantera, L; y Cervantes, G. (2010). Cuestionario de bienestar laboral general: Estructura y propiedades psicométricas. Revista de Psicología y Trabajo de las Organizaciones. 26(2), 157-170. Recuperado de https://bit. ly/2KxnXxo

- Comisión Europea. (1999). Guía sobre el estrés relacionado con el trabajo ¿La «sal de la vida» O el «beso de la muerte»? Dirección General de Empleo y Asuntos Sociales. Recuperado de https:// bit.ly/ 1ifigAD

- Congreso de Colombia. Ley 115 de 1994. Recuperado de https://bit.ly/2ITUJho

- Cornfield, D. B. (2006). Tendencias mundiales recientes en la sociología del trabajo. En: De la Garza, E. (Coord.). Tratado Latinoamericano de Sociología. P.122-132. Barcelona: Anthropos.

- Declaración de Bolonia. (1999). Recuperado de http://www.mat.ucm.es/ arrondo/Declaracion_ Bolonia.pdf

- Hernández Sampieri, R; Fernández Collado, C; y Baptista Lucio, P. (2014). Metodología de la investigación. 6 ed. México: McGraw-Hill.

- López, A. (sf). La Nueva Gestión Pública: Principios Teóricos e Implicancias Prácticas en el Quehacer de la Administración Estatal. Recuperado de https://bit.ly/2W9TrMx

- Ministerio de la Protección Social y Pontificia Universidad Javeriana. (2010). Batería de instrumentos para la evaluación de factores de riesgos psicosocial. Manual general. Bogotá: Ministerio de la Protección Social. Recuperado de https://bit. ly/20Mb099

- Ministerio del Trabajo y Organización Iberoamericana de Seguridad Social. (2015). Segunda encuesta nacional de condiciones de seguridad y salud en el trabajo en el sistema general de riesgos laborales de Colombia. Bogotá: Ministerio del Trabajo. Recuperado de https://bit.ly/2ZOWVTc 
- Ministerio del Trabajo. (2007). Primera encuesta nacional de condiciones de salud y trabajo en el sistema general de riesgos profesionales. Bogotá: Ministerio del Trabajo. Recuperado de https://bit. ly/2FxE5MB

- Ministerio del Trabajo. Decreto 1477 de 2014. Recuperado de https://bit.ly/2IOLbeP

- Oliveira, D. A; Gonçalves, G. B; y Melo Savana. D. G. (2004). Cambios en la organización del trabajo docente. Consecuencias para los profesores. Revista Mexicana de Investigación Educativa, 9(20), 183-197. Recuperado de https://bit.ly/2XqbWOD

- Organización Internacional del Trabajo. Resumen ejecutivo. (2017). Perspectivas Sociales y del Empleo en el Mundo 2017. Empresas y empleos sostenibles: empresas formales y trabajo decente. Ginebra: Organización Internacional del Trabajo. Recuperado de https://bit.ly/2Wogdos

- Organización Internacional del Trabajo. (2016). Estrés en el trabajo: un reto colectivo. Turín: Centro Internacional de Formación de la OIT. Recuperado de https://bit.ly/1N72DGD

- Ramírez González, D. A. y Soler Gaona, V. E. (2015). Condiciones de trabajo: control y autonomía sobre el trabajo y relaciones con jefes y directivos asociados al estrés laboral en docentes de algunas instituciones educativas de Colombia. Manizales. Trabajo de Grado. (Magíster en Gerencia del Talento Humano). Universidad de Manizales. Recuperado de https://bit.ly/2ESfIIV
- Ramírez, R.; Abreu, J. L.; y Badii, M. H. (2008). La motivación laboral, factor fundamental para el logro de objetivos organizacionales: Caso empresa manufacturera de tubería de acero. Revista Daena: International Journal of Good Conscience. 3(1), 143-185. Recuperado de http://www.spentamexico.org/v3-n1/3(1)\%20143-185.pdf

- Salanova, M. y Schaufelli, W. B. (2009). El engagement en el trabajo. Cuando el trabajo se convierte en pasión. Madrid: Alianza.

- Segurado Torres, A. y Agulló Tomás, E. (2002). Calidad de vida laboral: hacia un enfoque integrador desde la Psicología Social. Revista Psicothema. 14(4), 828-836. Recuperado de http://www.psicothema.com/pdf/806.pdf

- Useche, M. C. y Parra Queipo, B. (2002). La organización del trabajo en el marco de la globalización. Revista Gaceta Laboral. 8(1), 67-78. Recuperado de http://www.redalyc.org/articulo.oa?id=33608103

- Vieytes, R. (2009). Campos de aplicación y decisiones de diseño en la investigación cualitativa. En: Merlino, A. (Coord.). Investigación cualitativa en ciencias sociales. Temas, problemas y aplicaciones. (pp. 42-84). Buenos Aires: Cengage Learning. 